\title{
Optimización del proceso de aporte de recubrimientos anti- corrosión de Stellite 6 producidos mediante plaqueado láser $r^{(*)}$
}

\author{
I. Vicario*, C. Soriano*, C. Sanz ${ }^{*}$ R. Bayón ${ }^{*}$ y J. Leunda*
}

Resumen

\begin{abstract}
El plaqueado láser es una de las tecnologías de tratamiento superficial emergentes en el marco industrial actual. Se basa en el empleo de un haz láser para producir la fusión de un material de aporte sobre la superficie de un substrato en movimiento cuyas características se han de mejorar, consiguiéndose una unión de tipo metalúrgico entre ambos materiales, una baja porosidad y una reducción de la zona afectada térmicamente, en comparación con otras técnicas convencionales como el plasma de arco transferido (PTA), soldadura TIG, etc. Existen tres grandes campos de aplicación de este proceso: la reparación-restauración de componentes dañados, la producción de recubrimientos anticorrosión o anti-desgaste y, por último, el campo del prototipado rápido (fabricación directa por adición de material). En este trabajo se presenta un estudio del proceso de aporte de material mediante la técnica de plaqueado láser para producir recubrimientos anti-corrosión empleando aleación de Co Stellite 6 en polvo sobre un substrato de acero C45 (AISI 1045). Posteriormente, se lleva a cabo un estudio de las características frente a la corrosión del recubrimiento final obtenido y de su correlación con los parámetros de aporte empleados.
\end{abstract}

\section{Optimization of the deposition process of corrosion resistant Stellite 6 coatings produced by laser cladding}

Abstract

Keywords

\begin{abstract}
Laser cladding is one of the most efficient surface treatment technologies in the industry. It uses a laser heat source to deposit a thin layer of a desired material on a moving substrate, whose properties have to be improved, achieving a metallurgical bonding between them with low heat affected zone and low dilution, compared to other conventional technologies such as PTA, TIG welding or thermal Spraying. In this sense, it is remarkable that there are 3 main application fields for laser cladding technology: restoration or refurbishment of damaged parts, surface coating against corrosion or wear, and rapid prototyping. The present work describes a study of the optimization of the laser cladding of Co based coatings (Diamalloy 4060NS) on medium carbon steel C45 (AISI 1045). After laser treatment, the surface of the substrate material is improved in terms of resistance against corrosion; this is confirmed in the analysis performed afterwards. It is also shown that the corrosion barrier properties have direct correlation with the laser cladding variables.
\end{abstract}

Laser cladding; Corrosion; Stellite 6.

\section{INTRODUCCIÓN}

El proceso de plaqueado láser (laser cladding) consiste en la fusión de un material de aporte, generalmente en forma de polvo metálico, sobre la superficie de un material por la acción de un haz láser, con el fin de generar un recubrimiento de propiedades similares o diferentes a las del substrato ${ }^{[1]}$. Las ventajas del método incluyen una mínima dilución, baja y controlable entrada de calor en el substrato, gran flexibilidad de procesado y reducida distorsión ${ }^{[2}$ y 3$]$. Normal- mente, para llevar a cabo el proceso se emplean fuentes láser de $\mathrm{CO}_{2}$ y de $\mathrm{Nd}$ :YAG en régimen de funcionamiento continuo, si bien, en los últimos años, se están empleando las nuevas fuentes de diodos (HPDL), disco y fibra de alta potencia, así como láseres pulsados, con duraciones de pulso del orden de varios milisegundos ${ }^{[4-6]}$. Comparado con otras técnicas convencionales de deposición, como, soldadura TIG (Tungsten Inert Gas), proyección por arco de plasma o proyección HVOF (High Velocity Oxygen Fuel), la técnica de plaqueado láser produce cordones

\footnotetext{
(•) Trabajo recibido el día 31 de enero de 2007 y aceptado en su forma final el día 1 de octubre de 2008.

* Fundación Tekniker, Departamento de Procesos de Fabricación, Avda. Otaola 20. CP 20600, Eibar, España.
} 
de soldadura o recubrimientos libres de poros, grietas y deformaciones, con una buena unión metalúrgica con el substrato.

Stellite 6 es una de las aleaciones de base cobalto más común, empleada en la fabricación y reparación de componentes, como partes de turbinas de gas (álabes, estátores, etc.), componentes de automóvil (asientos de válvulas, cigüeñales, etc.)..., gracias a su alta dureza y buena resistencia frente a la corrosión y al desgaste. De hecho, las propiedades tribológicas, mecánicas y estructurales de recubrimientos de Stellite 6, generados con la técnica de plaqueado láser, sobre diferentes tipos de substratos: aceros inoxidables, al carbono, etc., ha sido estudiada en los últimos años por distintos autores ${ }^{[7-10]}$.

En el presente trabajo, se presenta un estudio del proceso de deposición de Stellite 6 sobre un substrato de acero C45 (AISI 1045) mediante la técnica de plaqueado láser. El trabajo se centra en el estudio de la influencia de los parámetros más relevantes del proceso a través del análisis de la geometría, dilución, micro-dureza y estructura de los recubrimientos generados. Adicionalmente, se ha estudiado el comportamiento frente a corrosión electroquímica en medio salino de los recubrimientos desarrollados.

\section{MÉTODO EXPERIMENTAL}

Para el desarrollo de la experimentación se empleó un generador láser de Nd:YAG, modelo DY022 de RofinBaasel, bombeado por diodos y emisión en modo continuo $(\mathrm{cw})$, con una potencia máxima de $2.200 \mathrm{~W}$. El haz láser fue guiado a la zona de trabajo a través de una fibra óptica de $600 \mu \mathrm{m}$ de diámetro, acoplada a un cabezal de aporte de polvo de Precitec (YC50), al cual se puede adaptar diferentes juegos de boquillas, según sea la anchura de los cordones de soldadura a generar. El cabezal del láser estaba sujeto a un robot ABB modelo IRB 4400, de 6 ejes de movimiento. Las partículas metálicas fueron dirigidas a través del cabezal láser coaxial por medio de un alimentador de polvo, modelo TWIN-10-C de Sulzer-Metco.

Por otra parte, se emplearon como material base probetas de sección rectangular $(120$ × 66 × $19 \mathrm{~mm})$ de acero $\mathrm{C} 45$, cuya composición en porcentaje en peso se detalla en la tabla I. El C45 es un acero no aleado y de un contenido medio de carbono. Se emplea, comúnmente, en piezas que necesitan una resistencia media-alta, como engranajes, piñones, cigüeñales, etc. Antes del endurecimiento, el material estaba normalizado, con una dureza en torno a $238 \mathrm{HV}(20 \mathrm{HRc})$.

Como material de aporte se empleó polvo de aleación de base cobalto, Stellite 6 (Diamalloy 4060NS) de Sulzer-Metco, con un tamaño de partícula entre 38
Tabla I. Composición nominal del acero al
carbono C45

Table I. Nominal composition of the C45 carbon steel

\begin{tabular}{cccc}
\hline C & Fe & Mn & S \\
\hline 0,5 & Bal. & 0,8 & 0,1 \\
\hline
\end{tabular}

y $106 \mu \mathrm{m}$. La composición en porcentaje en peso se muestra en la tabla II.

La experimentación consistió en la generación de diferentes cordones individuales sobre el substrato, variando dos de los parámetros más importantes que influyen en el proceso: la potencia del haz láser, en el intervalo de 1.000 a $2.200 \mathrm{~W}$ con un paso de $400 \mathrm{~W}$ y el caudal de polvo, de 2 a $30 \mathrm{~g} / \mathrm{min}$ con un paso de $2 \mathrm{~g} / \mathrm{min}$. La velocidad de avance del cabezal láser y el diámetro del haz desenfocado sobre la superficie del substrato se mantuvieron constantes, a $8,3 \mathrm{~mm} / \mathrm{s}$ y $2,2 \mathrm{~mm}$, respectivamente. Se empleó argón, a una presión fija de 5,0 bar, como gas de protección de las ópticas y propulsor de las partículas metálicas Seguidamente, se realizaron varios ensayos de cordones solapados, variando el grado de solape, con los parámetros que mejor resultado mostraron en la primera tanda $(1.000$ y $1.400 \mathrm{~W}$ a $14 \mathrm{~g} / \mathrm{min})$ en cuanto a buena adherencia, reducida dilución y ausencia de poros y grietas.

Las probetas se cortaron, pulieron y trataron químicamente para el estudio metalográfico. La geometría de los cordones se caracterizó mediante microscopia óptica. Un micro-durómetro Shimadzu modelo $\mathrm{M}$ se empleó para la medida de la micro-dureza en los cordones y en la zona afectada por el calor generados sobre el substrato.

Para estudiar el comportamiento frente a corrosión, las superficies recubiertas fueron previamente rectificadas y pulidas hasta conseguir una rugosidad similar a la del substrato $(0,5 \mu \mathrm{m})$. Los ensayos se realizaron en una celda electroquímica de tres electrodos compuesta por un electrodo de referencia de

\section{Tabla II. Composición nominal de la aleación Diamalloy $4060 \mathrm{NS}$}

Table II. Nominal composition of the Diamalloy 4060NS alloy

\begin{tabular}{cccccccc}
\hline C & Co & $\mathrm{Cr}$ & $\mathrm{Fe}$ & $\mathrm{Mo}$ & $\mathrm{Ni}$ & $\mathrm{Si}$ & $\mathrm{W}$ \\
\hline 1,2 & Bal. & 29,1 & 2,3 & 0,3 & 2,4 & 1,3 & 4,5 \\
\hline
\end{tabular}


$\mathrm{Ag} / \mathrm{AgCl}(\mathrm{KCl} 3 \mathrm{M})$ y un electrodo auxiliar de platino. Para simular el medio agresivo, se empleó un electrolito de $\mathrm{NaCl} 3,5 \%$ (pH 6,1). Tras aproximadamente dos horas de inmersión, se realizó una polarización lineal sobre cada muestra. El rango de potencial seleccionado varió desde $-0,2 \mathrm{~V}$ vs $\mathrm{Ag} / \mathrm{AgCl}$ hasta $+0,5 \mathrm{~V}$ vs $\mathrm{Ag} / \mathrm{AgCl}$ respecto al potencial a circuito abierto. $\mathrm{La}$ velocidad de barrido fue de $0,05 \mathrm{mV} / \mathrm{s}$.

\section{RESULTADOS}

\subsection{Geometría y micro-estructura}

La geometría de los cordones individuales generados se ha estudiado en términos de la relación de aspecto y la dilución. La relación de aspecto viene determinada mediante el cociente entre la anchura y la altura de cada uno de los cordones individuales. En la figura 1 se representa la relación de aspecto de los cordones en función de la potencia del láser suministrada y el caudal de polvo propulsado a través del alimentador.

Tal y como se muestra en la figura 1, el cociente anchura/altura de los cordones individuales generados disminuye conforme aumenta el caudal de polvo. Dado que la anchura del cordón se mantiene fija, al incidir un mayor número de partículas en un instante y área determinados, la energía funde mayor cantidad de estas partículas aumentando, así, la altura del cordón. A partir de un caudal de $10 \mathrm{~g} / \mathrm{min}$, la relación de aspecto se mantiene prácticamente constante para cada valor de potencia.

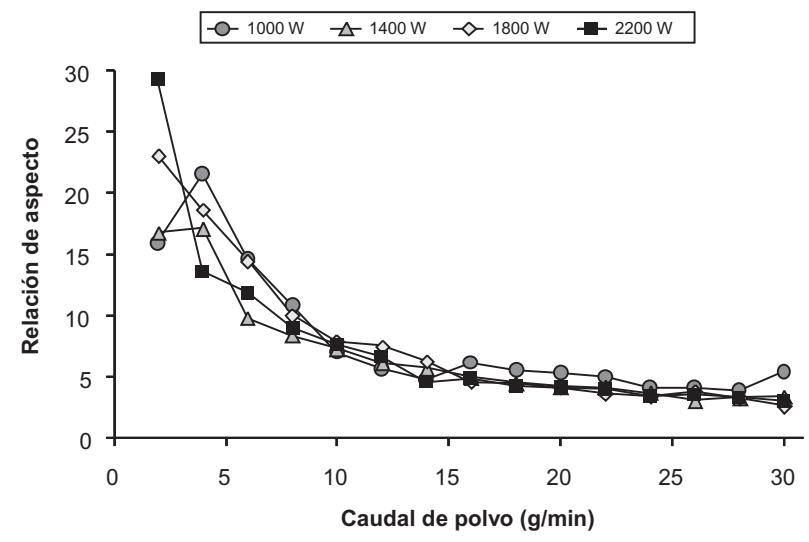

Figura 1. Relación de aspecto de los cordones individuales generados en función de la potencia para varios valores de caudal de polvo.

Figure 1. Aspect ratio of the single tracks generated depending as function of the power and powder mass flow rate.
Los cordones de soldadura obtenidos presentan buena apariencia, sin grietas ni discontinuidades. En la figura 2 se muestra la sección transversal de dos cordones individuales generados. En las imágenes puede apreciarse, claramente, el cordón de soldadura, la dilución, así como la zona afectada por el calor. La dilución es el parámetro geométrico que determina el grado de penetración del material aportado dentro del substrato. Viene definida en \%, como el cociente entre el área del cordón introducido dentro del substrato y el área total del cordón. De esta forma, la dilución del recubrimiento de la figura 2 a) es superior a la de la figura $2 \mathrm{~b}$ ).

En la figura 3 se representa la influencia del caudal de polvo y la potencia del haz láser sobre la dilución de los cordones. Los valores de dilución obtenidos son bastante elevados para todas las potencias, excepto a $1.000 \mathrm{~W}$, donde a partir de un caudal de polvo de $10 \mathrm{~g} / \mathrm{min}$, la dilución se mantiene por debajo del $3 \%$. Para un valor de potencia determinado, conforme aumenta el caudal de polvo disminuye el grado de dilución. Este efecto se debe, principalmente, a la atenuación que sufre el haz debido al mayor número de partículas metálicas incidentes lo que disminuye el número de partículas fundidas sobre la superficie del substrato.
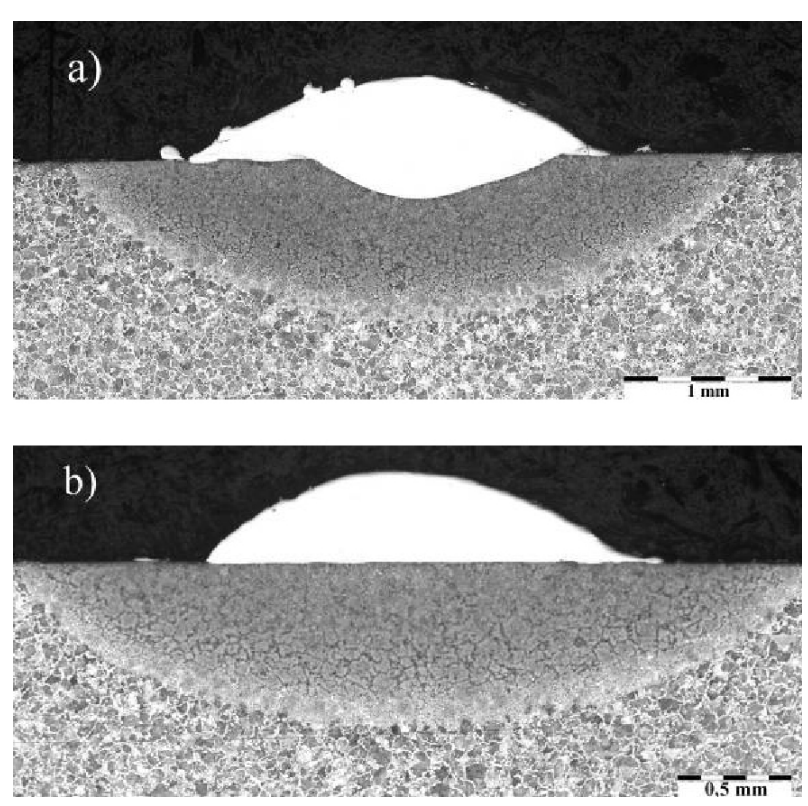

Figura 2. Sección transversal de dos cordones individuales, con valores de dilución diferentes; a) $36,6 \%$ a 1.400 W y $14 \mathrm{~g} / \mathrm{min}$; b) $2,4 \%$ a 1.000 W y $14 \mathrm{~g} / \mathrm{min}$.

Figure 2. Cross section of two single tracks with different dilution values; a) 36,6\% a 1,400 Wy $14 \mathrm{~g} / \mathrm{min}$; b) $2,4 \%$ a $1,000 \mathrm{~W}$ y $14 \mathrm{~g} / \mathrm{min}$. 


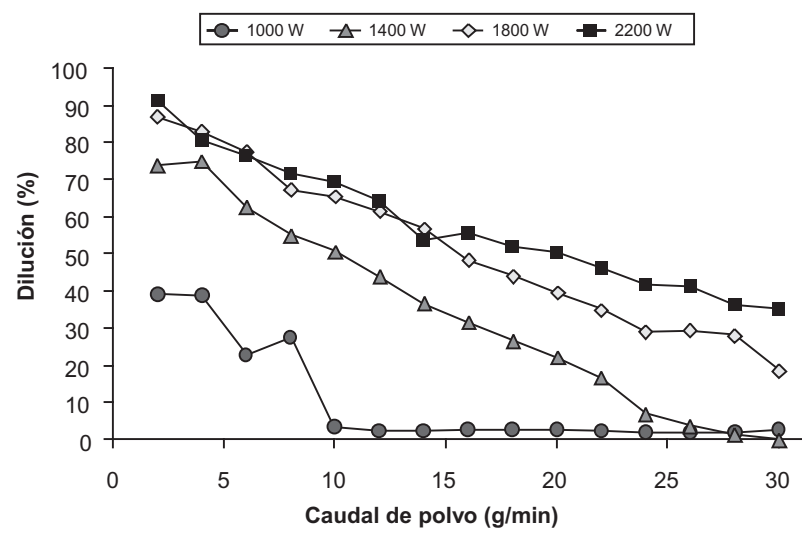

Figura 3. Dilución de los cordones individuales generados en función de la potencia para diferentes valores de caudal de polvo.

\section{Figure 3. Dilution of the single laser tracks generated as function of laser power and powder mass flow rate.}

Respecto a la micro-estructura, todos los cordones examinados presentan una estructura similar, formada por carburos en matriz dendrítica de solución sólida. La figura 4 a) refleja la estructura descrita. La zona afectada por el calor está formada por martensita, tal como se muestra en la figura $4 \mathrm{~b}$ ).

\subsection{Micro-dureza}

Los valores medios de micro-dureza de cada uno de los cordones obtenidos, expresados en HV1, en función del caudal de polvo y de la potencia del haz láser, están representados en la figura 5 . En ella, se puede observar como la micro-dureza presenta un valor máximo en torno a $800 \mathrm{HV} 1$, para caudales inferiores a $10 \mathrm{~g} / \mathrm{min}$, dependiendo del valor de la potencia. Seguidamente, la micro-dureza disminuye considerablemente hasta un valor mínimo entre 250 y 350 HV1 para, posteriormente, aumentar progresivamente con el caudal de polvo. Estos altibajos iniciales de la micro-dureza se pueden explicar, teniendo en cuenta que a valores de caudal tan bajos, prácticamente, no se genera cordón de soldadura produciendo que la mayor parte de la potencia del haz sea absorbida por el substrato, endureciendo la superficie del mismo.

El perfil de micro-durezas a través de la sección transversal de los dos cordones individuales de la figura 2 se muestra en la figura 6. Los valores medios de micro-dureza obtenidos fueron 459 y $548 \mathrm{HVO}, 3$ para los recubrimientos con 1.400 y $1.000 \mathrm{~W}$ de potencia, respectivamente. $\mathrm{Al}$ igual que se demuestra en la figura 5, la micro-dureza, en la región del recubrimiento
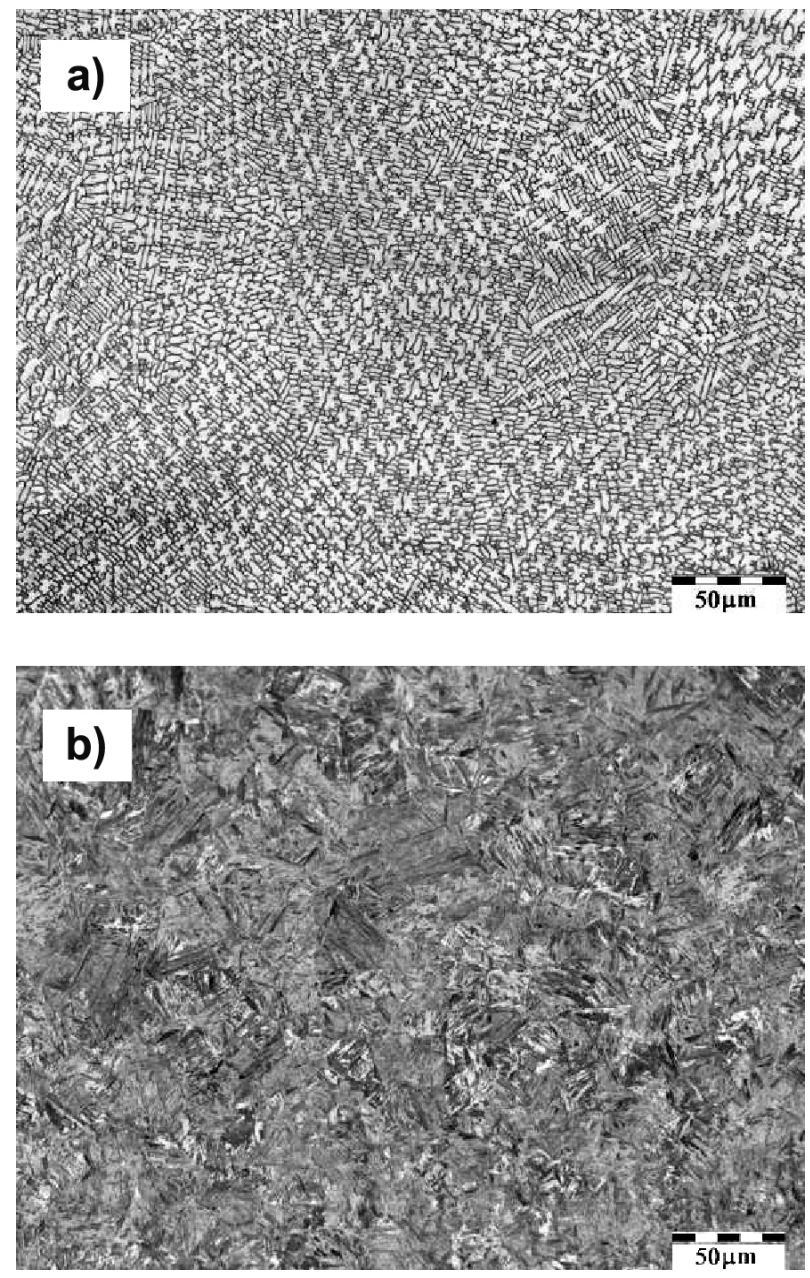

Figura 4. a) Detalle de la estructura dendrítica de un recubrimiento individual $(1.400 \mathrm{~W}, 14 \mathrm{~g} / \mathrm{min})$; b) Micrografía de la región afectada por el calor.

Figure 4. a) Detail of the dendritic structure of a single clad (1,400 W, $14 \mathrm{~g} / \mathrm{min}) ;$ b) Micrograph of the heat affected zone.

aumenta conforme la potencia disminuye y, por tanto, a la vista de los resultados, al disminuir la dilución.

Por otra parte, la dureza en la zona afectada por el calor es superior a la dureza obtenida por el recubrimiento, obteniéndose valores medios de $640 \mathrm{HV} 0,3$ para $1.400 \mathrm{~W}$ y $616 \mathrm{HV} 0,3$ para $1.000 \mathrm{~W}$ de potencia. Este efecto, es debido, principalmente, a la excelente capacidad de temple del material base empleado.

\subsection{Resistencia a la corrosión}

Las curvas de polarización se midieron sobre los siguientes recubrimientos: El primero de ellos, de cordones solapados al $25 \%, 1.000 \mathrm{~W}, 14 \mathrm{~g} / \mathrm{min}$ y 8,3 $\mathrm{mm} / \mathrm{s}$. El segundo, solapando cordones al $33 \%$, 


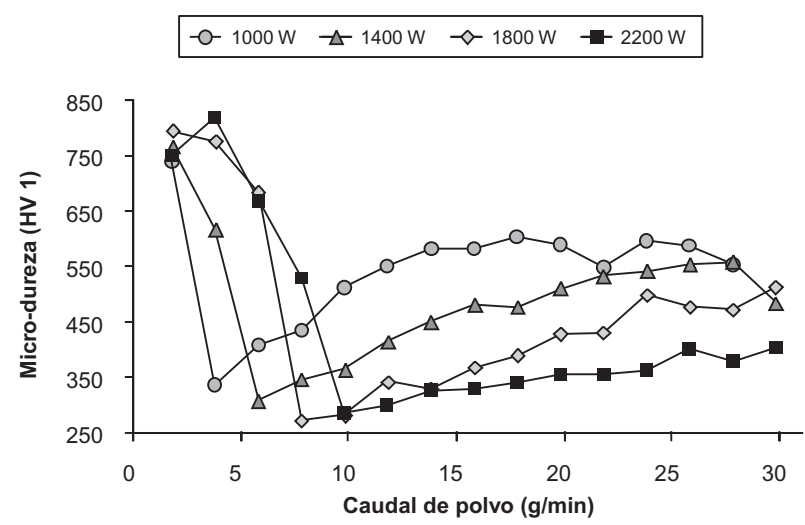

Figura 5. Micro-dureza HV1 de los cordones individuales depositados sobre acero C45 en función de la potencia láser para diferentes valores de caudal de polvo.

Figure 5. Micro-hardness HV1 of the single clads deposited on C45 steel as a function of the laser power for different powder mass flow rate values.

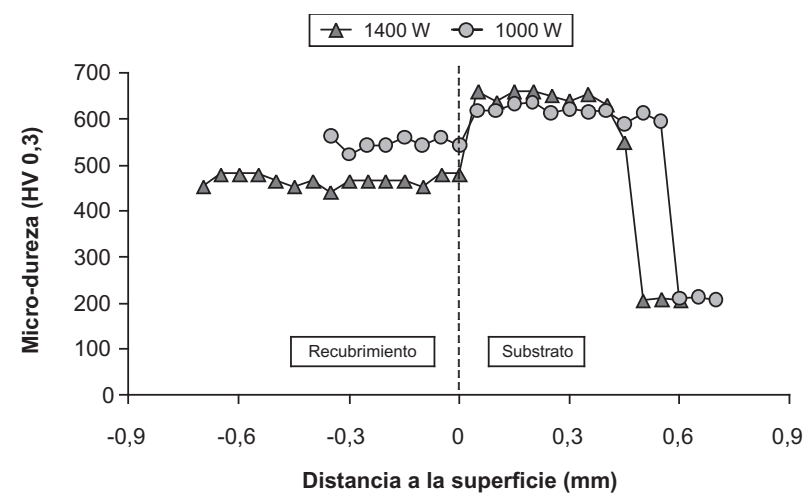

Figura 6. Perfil de micro-dureza de la sección transversal de los recubrimientos individuales de Stellite 6 generados sobre acero C45.

Figure 6. Micro-hardness profile across single laser Stellite 6 clads generated over C45 steel.

$1.400 \mathrm{~W}, 14 \mathrm{~g} / \mathrm{min}$ y $8,3 \mathrm{~mm} / \mathrm{s}$. Un ejemplo de este último se muestra en la figura 7 .

En la figura 8 se pueden observar las curvas de polarización registradas para ambos recubrimientos, así como la curva correspondiente al substrato. Ambos recubrimientos presentan un comportamiento pasivo en prácticamente todo el rango de potenciales anódicos abarcado, con corrientes de corrosión del orden de los $\mu \mathrm{A}$. El segundo recubrimiento, depositado a $1.400 \mathrm{~W}$, muestra una corriente ligeramente menor y un potencial de corrosión más noble. Los parámetros cinéticos obtenidos a partir del correspondiente análisis de Tafel, se muestran en la tabla III.

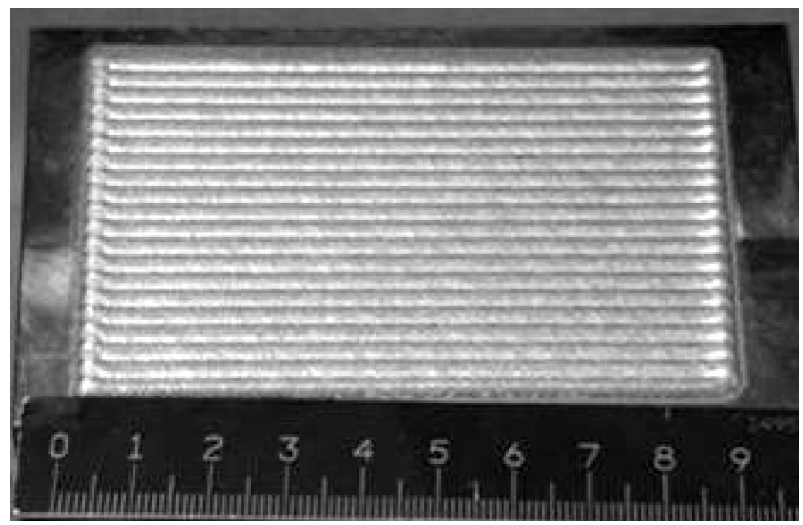

Figura 7. Apariencia exterior de un recubrimiento formado por 20 cordones solapados al $33 \%$ (1.400 W, $14 \mathrm{~g} / \mathrm{min})$.

Figure 7. External appearance of a laser cladding composed of 20 single clads overlapped at $25 \%$ $(1,400 \mathrm{~W}, 14 \mathrm{~g} / \mathrm{min})$.

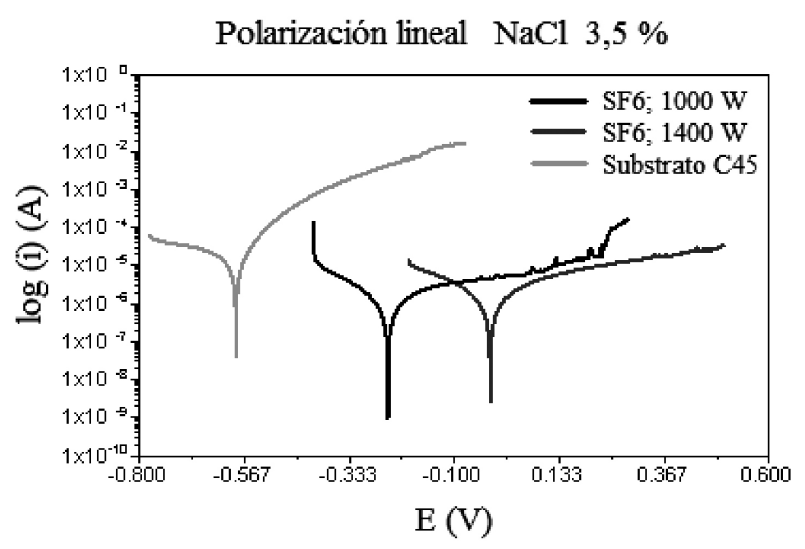

Figura 8. Potenciales de corrosión electroquímicos en función de la corriente para el material base $\mathrm{C} 45$ y los recubrimientos de Stellite 6 a 1.000 y $1.400 \mathrm{~W}$.

Figure 8. Electrochemical corrosion potentials as function of the current for $C 45$ base material and the Stellite 6 coatings at 1,000 and 1,400 W.

Tabla III. Resultados cinéticos obtenidos tras el análisis de Tafel

Tabla III. Kinetics results obtained after the Tafel analysis

\begin{tabular}{lcc}
\hline \multicolumn{1}{c}{ Material } & $\begin{array}{c}\mathrm{I}_{\text {corr }} \\
\left(\boldsymbol{\mu} \mathrm{A} / \mathrm{cm}^{2}\right)\end{array}$ & \\
\hline SF6; $1000 \mathrm{~W}$ & 2,62 & $\mathrm{E}_{\mathrm{cor} \mathrm{r}}$ \\
SF6; 1400 W & 2,09 & $-0,019$ \\
Substrato C45 & 14,95 & $-0,584$ \\
\hline
\end{tabular}




\section{CONCLUSIONES}

En este trabajo se ha presentado un estudio del proceso de plaqueado láser de la aleación de base Co Stellite 6 sobre un substrato de acero al carbono C45.

Se ha estudiado y discutido las características de los recubrimientos generados según la relación de aspecto, dilución, micro-estructura, micro-dureza y resistencia a la corrosión, obteniéndose los siguientes resultados:

- Se han obtenido recubrimientos individuales y solapados con buena adherencia, apariencia exterior, baja dilución, libres de grietas y porosidades.

- La relación de aspecto de los recubrimientos disminuye conforme aumenta el caudal de polvo manteniéndose prácticamente constante a partir de $10 \mathrm{~g} / \mathrm{min}$, para cada uno de los valores de potencia explorados.

- El parámetro de dilución disminuye con el aumento del caudal de polvo y aumenta con la potencia. Se han obtenido valores inferiores al $5 \%$ a partir de $10 \mathrm{~g} / \mathrm{min}$ con una potencia de $1.000 \mathrm{~W}$.

- La micro-estructura de todos los recubrimientos generados muestra una matriz dendrítica, similar para todos los recubrimientos generados.

- La micro-dureza de los recubrimientos de Stellite 6 disminuye conforme aumenta la potencia del haz láser y aumenta con el caudal de polvo. Se han obtenido valores de dureza ligeramente superiores en la zona afectada por el calor con respecto al recubrimiento, con una diferencia media de $75 \mathrm{HV}$, dada la capacidad de endurecimiento del acero C45.

- Los resultados de los ensayos de corrosión confirman un excelente comportamiento frente a corrosión de los recubrimientos analizados.
Ambos recubrimientos ofrecen al substrato una alta protección frente a corrosión en el medio agresivo estudiado.

\section{Agradecimientos}

El trabajo desarrollado en este artículo se ha llevado a cabo en el marco del proyecto titulado "Aplicaciones industriales de la tecnología láser: desarrollo de demostrador para máquina multifuncional"-Lamiak. Dicho proyecto fue subvencionado por la Diputación Foral de Guipúzcoa y cofinanciado por la Unión Europea, con Fondos Feder.

\section{REFERENCIAS}

[1] E. Toyserkani, A. Khajepour y S. Corbin, Laser Cladding, CRC Press, Boca Raton, Florida, EE. UU., 2005, pp. 1-22.

[2] R. Vilar, Mater. Sci. Forum. 301 (1999) 229252.

[3] U. De Oliveira, V. Ocelik y J.Th.M De Hosson, Surf. Coat. Technol. 197 (2005) 127-136.

[4] Y.P. Kathuria, Surf. Coat. Technol. 132 (2000) 262-269.

[5] A. Conde, F. Zubiri y J. de Damborenea, Mater. Sci. Eng. A 334 (2002) 233-238.

[6] S. Sun, Y. Durandet y M. Brandt, Surf. Coat. Technol. 194 (2005) 225-231

[7] J.Th.M de Hosson y L. De Mol Van Otterloo, Surf. Eng 13 (1997) 471-481.

[8] C. Navas, A. Conde, M. Cadenas y J. de Damborenea, Surf. Eng. 22 (2006) 26-34.

[9] H. So, C.T. Chen y Y.A. Chen, Wear 192 (1996) 78-84

[10] L. Mingxi, H. Yizhu Y S. Guoxiong, Appl. Surf. Sci. 230 (2004) 201-206. 